\title{
The Running Coupling from SU(3) Potentials
}

\author{
UKQCD Collaboration - presented by Chris Michael ${ }^{\text {a }}$ \\ ${ }^{a}$ DAMTP, University of Liverpool, Liverpool L69 3BX, UK
}

\begin{abstract}
From an accurate determination of the inter-quark potential, one can study the running coupling constant for a range of $R$-values and hence estimate the scale $\Lambda_{\overline{\mathrm{MS}}}$. Detailed results are presented for $S U(3)$ pure gauge theory from a study of a $36^{4}$ lattice at $\beta=6.5$.
\end{abstract}

\section{Introduction}

In the continuum the potential between static quarks is known perturbatively to two loops in terms of the scale $\Lambda_{\overline{\mathrm{MS}}}$. For $S U(3)$ colour, the continuum force is given by [1]

$\frac{d V}{d R}=\frac{4}{3} \frac{\alpha(R)}{R^{2}}$

with the effective coupling $\alpha(R)$ given by

$$
\frac{1}{4 \pi\left[b_{0} \log \left(R \Lambda_{R}\right)^{-2}+\left(b_{1} / b_{0}\right) \log \log \left(R \Lambda_{R}\right)^{-2}\right]}
$$

where $b_{0}=11 / 16 \pi^{2}$ and $b_{1}=102 b_{0}^{2} / 121$ are the usual coefficients in the perturbative expression for the $\beta$-function and, neglecting quark loops in the vacuum, $\Lambda_{R}=1.048 \Lambda_{\overline{\mathrm{MS}}}$. Note that the usual lattice regularisation scale $\Lambda_{L}=0.03471 \Lambda_{\overline{\mathrm{MS}}}$.

At large separation $R$, the potential behaves as $K R$ where $K$ is the string tension. Thus in principle knowledge of the potential $V(R)$ serves to determine the dimensionless ratio $\sqrt{K} / \Lambda$ which relates the perturbative scale $\Lambda$ to a nonperturbative observable such as the string tension $K$. This is the basis of the method [2] used for $S U(2)$ which we extend here to $S U(3)$ colour.

For $S U(3)$ as for $S U(2)$, the bare lattice coupling proves to be a poor guide to physical behaviour in that asymptotic scaling to two loops is not yet manifest. However, the weaker requirement of scaling is well satisfied: the dimensionless ratios of physical quantities are found to be independent of $\beta$. For example in $S U(2)$, the potential $V(R)$ scales [3] over a range of lattice spacing of a factor of 4 (from $\beta=2.4$ to 2.85). That scaling but not asymptotic scaling is valid implies that the bare coupling constant derived from $\beta$ is inappropriate and that an effective coupling constant derived from some physical quantity is a better choice. This has been emphasized by Lepage and Mackenzie 㕶. It is also the basis of the method proposed by Lüscher et al. [5] to extract the running coupling constant.

Here we choose to determine the running coupling constant from the interquark potential between static quarks at small distance $R$. This quantity can be determined in a straightforward way from lattice simulation on large volume lattices. Although we require small $R$ and hence large energy $1 / R$ to make most precise contact with the perturbative expression, the lattice method implies the presence of lattice artefacts when $R \approx a$. Thus we need to work on the largest spatial lattice available, consistent with avoiding finite size effects. We present results from a $36^{4}$ lattice at $\beta=6.5$ to achieve this [6]. These results are compared with previous UKQCD data [7] from $24^{3} \times 48$ lattices at $\beta=6.2$ to check scaling.

\section{Lattice potentials}

Previous studies of glueballs and potentials in quenched $S U(3)$ have found evidence [8] for finite size effects when the spatial lattice size $L$ is such that $m L<9$ where $m$ is the $0^{++}$glueball mass. This corresponds to $\sqrt{K} L<2.6$. Our previous study on a $24^{3} \times 48$ lattice [7] at $\beta=6.2$ corresponded to $\sqrt{K} L=3.8$ which appears to be comfortably in the large volume region where finite size effects are negligible. For our study at larger $\beta$ we are constrained by the memory limitation to a $36^{4}$ lattice and we selected $\beta=6.5$ since 
this again corresponds to the same spatial size in physical units, namely $\sqrt{K} L=3.8$. Even though direct study of the potential seems to suggest [9] that it is relatively insensitive to finite size effects, we prefer to adopt the cautious policy of conducting our study at a fixed large physical volume.

The details of our analysis of the potential between static sources on these $36^{4}$ lattices are given elsewhere [6]. Here we summarise the main method and results. We conducted a relatively high statistics study of the potential at large $R$ to be able to get an accurate value of the string tension $K$. From a fit to $R$-values from $4 a$ to $24 a$ we obtain $K a^{2}=0.0114(4)$ where a systematic error from $T$-extrapolation is included. The values of the force are collected in table 1 .

In order to explore the potential at small $R$, we measure at off-axis as well as on-axis separations. This will allow us to measure the extent to which rotational invariance is broken down to the cubic invariance on the lattice. Furthermore, we find that a simple parametrisation in terms of a lattice one gluon exchange is able to parametrise successfully our results. This is non-trivial since there is only one free parameter to accommodate the breaking of rotational invariance and yet a satisfactory fit is obtained to all the off-axis and on-axis data. The details of the fitting procedure are exactly those pioneered successfully for $S U(2)$ [2].

From this successful parametrisation of the departure from rotational invariance, we can construct a corrected potential by replacing the lattice gluon contribution in the fit by the full continuum gluon propagator. These results are shown in table 2 .

\section{Running Coupling}

It is now straightforward to extract the running coupling constant by using

$\alpha\left(\frac{R_{1}+R_{2}}{2}\right)=\frac{3}{4} R_{1} R_{2} \frac{V_{c}\left(R_{1}\right)-V_{c}\left(R_{2}\right)}{R_{1}-R_{2}}$

where the error in using a finite difference is here negligible. This is shown in table 2 and is plotted in the figure versus $R \sqrt{K}$ where $K$ is taken from the large $R$ fit. The interpretation of $\alpha$ as defined
Table 1

The force $\Delta V / \Delta R$ at average separation $R$ derived from $T$-ratio $4 / 3$.

\begin{tabular}{rl}
\hline$R / a$ & \multicolumn{1}{c}{$\Delta V / \Delta R$} \\
\hline 2.4142 & $0.0667(4)$ \\
3.4142 & $0.0344(4)$ \\
4.2361 & $0.0286(8)$ \\
5.0645 & $0.0226(5)$ \\
5.8284 & $0.0196(19)$ \\
6.1623 & $0.0190(16)$ \\
6.7678 & $0.0174(9)$ \\
7.6056 & $0.0161(10)$ \\
8.2426 & $0.0161(11)$ \\
9.0000 & $0.0149(3)$ \\
11.0000 & $0.0130(4)$ \\
13.0000 & $0.0134(5)$ \\
15.0000 & $0.0121(5)$ \\
17.0000 & $0.0132(6)$ \\
19.0000 & $0.0117(6)$ \\
21.0000 & $0.0125(8)$ \\
23.0000 & $0.0126(7)$ \\
\hline
\end{tabular}

Table 2

The force $\Delta V / \Delta R$ and lattice artefact corrected force $\Delta V_{c} / \Delta R$ at average separation $R$. The running coupling $\alpha(R)$ derived from the corrected force is shown as well. The second error shown on $\alpha$ is $10 \%$ of the lattice artefact correction.

\begin{tabular}{clcl}
\hline$R / a$ & \multicolumn{1}{c}{$\Delta V / \Delta R$} & $\Delta V_{c} / \Delta R$ & \multicolumn{1}{c}{$\alpha(R)$} \\
\hline 1.2071 & $0.2067(7)$ & 0.1607 & $0.170(1)(5)$ \\
1.7071 & $0.0750(7)$ & 0.0930 & $0.197(1)(4)$ \\
2.1180 & $0.0959(19)$ & 0.0664 & $0.223(6)(10)$ \\
2.5322 & $0.0541(5)$ & 0.0523 & $0.248(2)(1)$ \\
2.9142 & $0.0263(40)$ & 0.0424 & $0.270(26)(10)$ \\
3.0811 & $0.0471(39)$ & 0.0368 & $0.262(28)(7)$ \\
3.3839 & $0.0391(12)$ & 0.0371 & $0.317(10)(2)$ \\
3.9241 & $0.0292(5)$ & 0.0290 & $0.333(6)(1)$ \\
\hline
\end{tabular}


above as an effective running coupling constant is only justified at small $R$ where the perturbative expression dominates. Also shown are the two-loop perturbative results for $\alpha(R)$ for different values of $\Lambda_{R}$.

Figure 1. The effective running coupling constant $\alpha(R)$ obtained from the force between static quarks at separation $R$. The scale is set by the string tension $K$. Data at $\beta=6.5$ are from table 2 (diamonds) and at $\beta=6.2$ (triangles). The dotted error bars represent an estimate of the systematic error due to lattice artefact correction as described in the text. The curves are the two-loop perturbative expression with $a(6.5) \Lambda_{R}=0.060$ (dotted) and 0.070 (continuous).

The figure clearly shows a running coupling constant. Moreover the result is consistent with the expected perturbative dependence on $R$ at small $R$. There are systematic errors, however. At larger $R$, the perturbative two-loop expression will not be an accurate estimate of the measured potentials, while at smaller $R$, the lattice artefact corrections are relatively big. Setting the scale using $\sqrt{K}=0.44 \mathrm{GeV}$ implies $1 / a(\beta=6.5)=4.13$ $\mathrm{GeV}$, so $R<4 a(6.5)$ corresponds to values of $1 / R>1 \mathrm{GeV}$. This $R$-region is expected to be adequately described by perturbation theory. Another indication that perturbation theory is accurate at such $R$-values is that $\Delta V_{c} / \Delta R$ at small
$R$ is found to be very much greater than the nonperturbative value $K$ at large $R$.

Even though the lattice artefact correction of all 6 off-axis points by one parameter is very encouraging, the only way to be certain that lattice artefacts are eliminated is by the comparison of different $\beta$ values (with thus different $R / a$ values at the same physical $R$ value). Now this test was satisfied in an $S U(2)$ study [2]3]. Even so we can check independently in $S U(3)$ and we use UKQCD data [7] from $24^{3} \times 48$ lattices at $\beta=6.2$. We follow the same procedure as above (for more details see [7]) and obtain for the string tension $K a^{2}=0.0251(8)$. This corresponds to a ratio of lattice spacings $a(6.2) / a(6.5)=1.484(35)$ to be compared with the two-loop perturbative ratio of 1.404. From the small $R$ results we then obtain a corrected force and hence a running coupling. Setting the scale from the measured string tensions, we also show the $\beta=6.2$ results for this effective running coupling in the figure. There is excellent agreement with the results from $\beta=6.5$.

The easiest way to describe the value of the running coupling constant $\alpha$ is in terms of a scale or $\Lambda$ value with the understanding that we are only determining $\alpha$ for a range of energy scales $1 / R$ - namely 1 to $3 \mathrm{GeV}$. The final estimate of $\Lambda$ is made from the figure, weighting smaller $R$ more heavily since the perturbative expression is more accurate as $\alpha(R)$ becomes smaller. We exclude the lowest $R$ point since the lattice artefact correction is for $R>a$. Remembering that the systematic errors due to lattice artefact correction are estimates only and since these systematic errors are dominant, we do not attempt a fit but we can conclude that our results are consistent with values of $\Lambda$ lying in the range shown by the two curves plotted. From the data at $\beta=$ 6.5 , these curves have $a(6.5) \Lambda_{R}=0.070$ and 0.060 . Using the value of the string tension from the fit, we get $\sqrt{K} / \Lambda_{L}=49.6(3.8)$. Moreover, this value is consistent with the evaluation at both $\beta=6.5$ and 6.2. A rather similar analysis by Bali and Schilling [9] also agrees with this result. 


\section{Conclusions}

Using the bare coupling $g$ derived from $\beta=$ $6 / g^{2}$ and the two-loop perturbative relationship $a(g)$ in terms of the scale $\Lambda_{L}$ gave [10,7,9] the following slowly decreasing values of $\sqrt{K} / \Lambda_{L}=$ 93.0(7) and 96.7(1.6)(2.6) at $\beta=6.0 ; 85.9(1.5)$ and 86.4(1.0)(1.9) at $\beta=6.2$ and 82.3(8)(1.7) at $\beta=6.4$. Our present analysis at $\beta=6.5$ yields $\sqrt{K} / \Lambda_{L}=80.0(1.4)$. Clearly, the $\beta \rightarrow \infty$ limit lies below these values. Moreover the statistically significant decrease of these values is evidence that two-loop perturbative scaling is not obtained in terms of the bare coupling. Our present method which does not rely on the bare coupling gives the scaling result which should be independent of $\beta$. Our estimate is $\sqrt{K} / \Lambda_{L}=$ 49.6(3.8). This is sufficiently far below the values extracted from the bare coupling to imply that asymptotic scaling to two-loop perturbation theory is not "just around the corner" but will only be satisfied accurately at larger $\beta$-values than those currently accessible to lattice simulation.

Using an empirical definition of an effective coupling constant in terms of the measured plaquette action, it is possible to extrapolate $\sqrt{K} / \Lambda_{L}$ to $a=0$ and the resulting estimates 11 , 9] are in agreement with our value. This provides additional support for the viewpoint that the bare lattice coupling has bad perturbative behaviour but that perturbative descriptions of small distance phenomena at existing $\beta$ values are reliable if a physical coupling definition is used.

Our result for the running coupling $\alpha_{R}(R)$ given in the figure and table 2 can be read directly as $\alpha_{\overline{\mathrm{MS}}}(q)$ with $q=1 / R$ since these schemes are so close to each other. Since we obtain results consistent with the perturbative evolution, we can estimate the continuum ratio $\sqrt{K} / \Lambda_{\overline{\mathrm{MS}}}=1.72(13)$ for pure $\mathrm{SU}(3)$ theory. Setting the scale using $\sqrt{K}=0.44 \mathrm{GeV}$, then gives $\Lambda_{\overline{\mathrm{MS}}}=256(20) \mathrm{MeV}$. These results are obtained for rather modest energies $(1 / R \approx 1-3 \mathrm{GeV})$ but there is evidence from studies in $S U(2)$ where higher energies have been reached [3] that the method is stable as the energy scale is increased somewhat. From lattice results for ratios of other non-perturbative quantities (glueball masses, critical temperature, etc) to the string tension, one can then determine their value in terms of $\Lambda_{\overline{\mathrm{MS}}}$ as well.

Even though the scales probed in this work are relatively small (i.e. only $3 \mathrm{GeV}$ ), the agreement with the perturbative evolution of the coupling constant implies that this is a reasonable way to determine the coupling constant in terms of nonperturbative physical quantities. We are able to determine $\Lambda_{\overline{\mathrm{MS}}}$ relatively accurately compared to experiment. Of course experiment has full QCD with dynamical light quarks included while precise lattice simulation of full QCD is still a considerable challenge.

\section{REFERENCES}

1. A. Billoire, Phys. Lett. 104B (1981) 472.

2. C. Michael, Phys. Lett. 283B (1992) 103.

3. S.P. Booth et al. (the UKQCD Collaboration), Phys. Lett. B 275 (1992) 424; Liverpool preprint LTH284.

4. P. Lepage and P. Mackenzie, Nucl. Phys. B (Proc. Suppl.) 20 (1991) 173.

5. M. Lüscher, P. Weisz and U. Wolff, Nucl. Phys. B359 (1991) 221;

M. Lüscher, R. Sommer, U. Wolff and P. Weisz , CERN preprint TH 6566/92, DESY preprint 92-096.

6. S. P. Booth et al. (the UKQCD Collaboration), Liverpool preprint LTH285; Phys. Lett. B (in press).

7. C. R. Allton et al. (the UKQCD Collaboration), Phys. Lett. B 284 (1992) 377 and in preparation.

8. C. Michael and M. Teper, Nucl. Phys. B 314 (1989) 347.

9. G. Bali and K. Schilling, Wuppertal preprints WUB 92-02; WUB 92-29 (1992).

10. S. Perantonis and C. Michael, Nucl. Phys. B 347 (1990) 854.

11. J. Fingberg, U. Heller and F. Kasrch, Bielefeld preprint BI-TP 92-26. 